\title{
“Long-term benefits of airway clearance in bronchiectasis: a randomised placebo- controlled trial." Gerard Muñoz, Javier de Gracia, Maria Buxó, Antonio Alvarez and Montserrat Vendrell. Eur Respir J 2018; 51: 1701926.
}

\section{CrossMark}

This article from the January 2018 issue of the European Respiratory Journal was originally published with errors in figure 1. The numbers of subjects for each institute indicated in the box headed "Centre distribution" should have been

Dr Josep Trueta Hospital $(\mathrm{n}=37)$ and

Vall d'Hebron Hospital $(n=7)$

and in the box headed "Allocated to placebo $(n=22)$ " should have been

Received allocated intervention $(\mathrm{n}=22)$

Dr Josep Trueta Hospital $(\mathrm{n}=19)$ and

Vall d'Hebron Hospital $(\mathrm{n}=3)$

The corrected figure is presented below, and the original article has been corrected and republished online. No further changes to the body text of the article were needed to address the error.

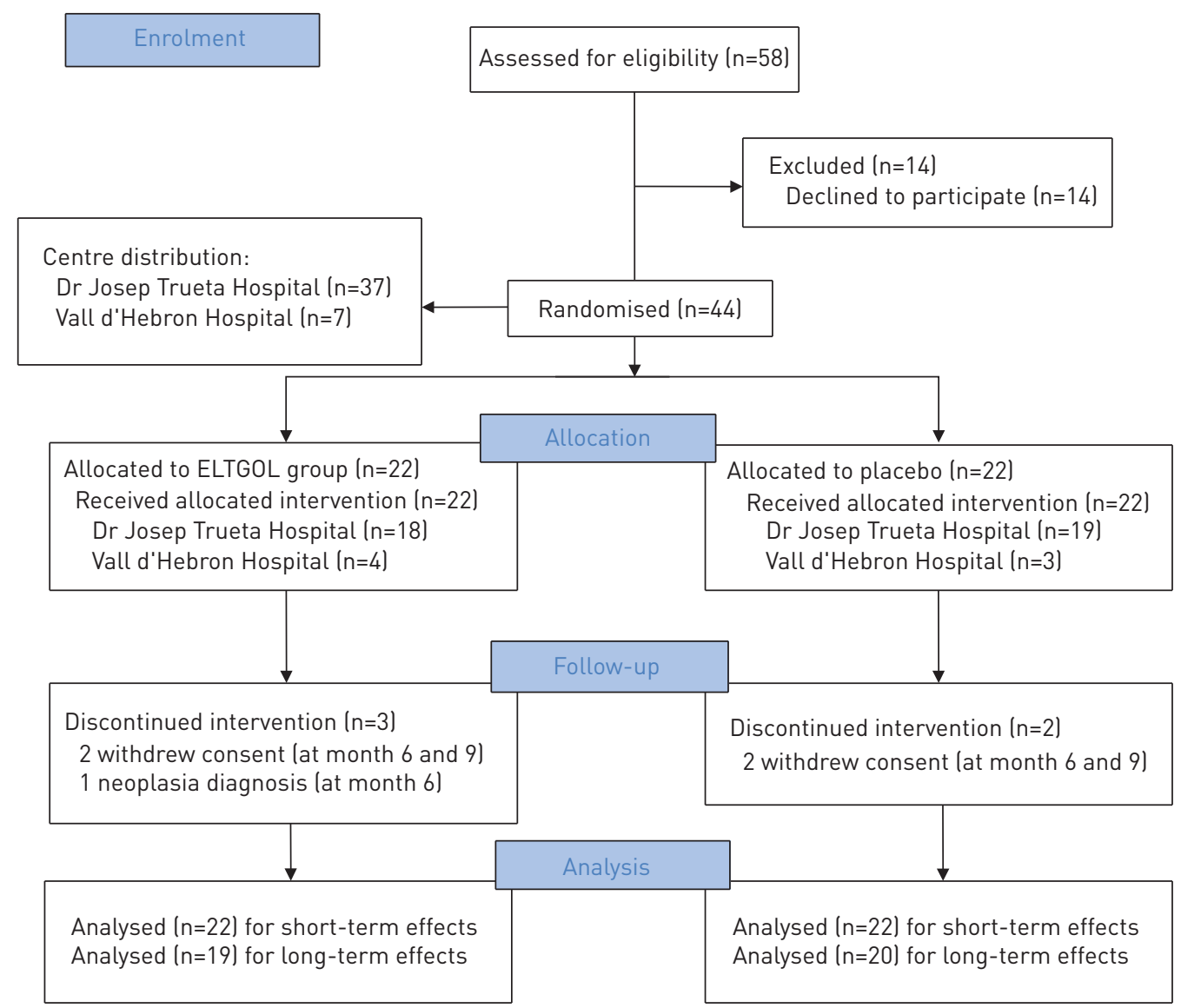

FIGURE 1 Trial profile. 